\title{
Exploring the Impact of Hostel Life of Students on Academic Performance: Sohar University - a case study Firdouse Rahman Khan ${ }^{1}$, Najat Said Hamad Alshekili², Aida Said Al Badi ${ }^{3}$ \& Hakeema Aamir Al Khanbashi ${ }^{4}$ \\ Faculty of Business, Sohar University, Sultanate of Oman \\ Email: ${ }^{1}$ firdouse4u@yahoo.co.uk, ${ }^{2}$ najatsaid907@gmail.com, ${ }^{3}$ aida@gmail.com, \\ hakeema.aamir@gmail.com
}

Article History: Received on $7^{\text {th }}$ Feb. 2020, Revised on $8^{\text {th }}$ Mar 2020, Published on $10^{\text {th }}$ May 2020

\begin{abstract}
Purpose of the study: The objective of the study is to critically investigate the facilities and the communication services required in the hostel; to critically investigate the safety services prevailing in the hostel.
\end{abstract}

Design/Methodology/Approach: For this study, 219 respondents were chosen on a simple random sampling basis among the students both girls and boys from Sohar University who were living in the hostel outside side the campus. Using a well-defined questionnaire, the data was collected data and analyzed using Statistical Package for Social Sciences (SPSS) to derive at the conclusion.

Findings: The empirical study shows that the students are satisfied with the Room Facilities and the Safety Services provided in the hostel. However, they are not satisfied with the Communication Services. The study also reveals that there is an impact of Communication Services and Family Support on Study Convenience for the students living in a hostel.

Practical Implications: The hostel authorities/administration should pay more attention to the communication facilities provided in the hostel especially speedy internet connection that could be made available through Wi-Fi to convene the students.

Social Implications: Hostel life is a principal component in the academic life of students who leave their home and live for learning. The factors which create an impact of hostel life on academic performance need to be paid attention by the educational authorities so that the ultimate purpose of students joining the institutions viz. learning can be achieved.

Originality/value: No study has investigated before the impact of hostel life on academic performance, and this study will help the College / University Administration to plan and provide the necessary facilities for the students.

Keywords: Students' Housing Facilities, Impact of hostel life on Academic Performance, Sohar University Students' Hostel life, Students' Satisfaction with the hostel facilities, Students' Study Convenience.

\section{Introduction}

Hostel life is a memorable moment for a student during his/her academic life period who will be living away from his/her home. The hostel can be described as an area occupied by a group of students who come together from far places for the common purpose of acquiring knowledge called learning. Usually, a hostel consists of the common facilities such as kitchen, bathroom, reading room, guest hall, reception area, recreation room, etc. Some universities provide students with such facilities within the campus and to some outside the campus. Indeed, it is a new experience for those young students who live in the hostels, far away from their families. This gives them a chance to learn how to live independently, gain adaptability to live with roommates and other students, share space utilities and solutions, etc.

In the bygone days, the hostel was considered as a punishment area to those students who were mischievous and considered to be similar to asylums. In the modern era, the hostel is considered to be suitable for students because it is free from the disturbances of families and related issues. It is considered that there are lots of advantages due to living in a hostel. A student may get help from his/her senior students in their studies and can borrow books from classmates, others, etc. There are great chances of learning in the form of group studies, exchanging books, magazines, and newspapers through roommates and other hostellers. A student might get to learn self-reliance, social behavior, independence and may learn how to manage his/her time, manage his/her residential room and spend money efficiently and concentrate on studies to increase his/her academic performance. 
As we know a hostel is an important place for the students, who come to live far away from their home leaving their families. Hostel life can be very much different for them compared to their life at home. Life in the hostel makes them depend upon themselves and make them smart, active, and disciplined too. However, it is an obvious question whether the hostel life makes an impact on the students' academic performance or not as their ultimate aim for staying in a hostel is to acquire knowledge and skills. So this study focuses on finding out the expectation and the satisfaction of the hostel life of the students and their impact thereby on the academic performance.

\section{Review of Literature}

Iftikar and Ajmal (2015) investigated the impact of hostel life found that the hostel has great importance in the educational journey and male students are more inclined to be affected harmfully. Owolabi (2015) investigated and found that there is a difference between the academic performance of students who were staying in on-campus and of those staying off-campus housing. Shakeel, Shakeel, and Fatima (2015) found that happiness in student's hostel life is essential for their learning aims, and their quality of life affects their learning. Khurshid, Tanveer, and Qasmi (2012) investigated the relationship between academic achievement and the students' habits from the hostel. Ning and Chen (2016) found that the university dormitories equipped with quality physical facilities support students' academics. Araujo and Murray (2010) confirmed that the result of dormitory living students' performance rise Grade Point Average (GPA) by 0.19 to 0.9. Mahama, Boahen, Saviour, and Tumaku (2016) investigated what should exist in an inexpensive hostel confirmed that a calm and peaceful environment with proper security, and water, toilet, and electricity facilities. Mansoor and Ali (2015) investigated the hostel life impact on academic performance found that the factors affecting student satisfaction were the basic amenities such as bathrooms and laundry with adequate water supply, kitchenette, and uninterrupted electricity supply. Kamaruddin, Zainal, and Aminuddin (2009) found that the four prime factors which support the students' academic performance are the environment, housing, facilities provided, and motivation. Khozaei, Ayub, Hassan, and Khozaei (2010) found the factors predicting student's satisfaction with hostel life are hostel fees, hostel security, size and safety of the room, distance from university facilities, hostel facilities and good internet network connection. Owusu (2013) revealed that the students were of the view that reading habits affect academic performances and also found that laziness is one of the basic hindrances to their reading habits. Owusu-Acheaw and Larson (2015) found that the students use their internet-enabled phones in their accommodations to complement their studies in the library. Veena (2015) found that most of the students were satisfied with the existing Wi-Fi facility in the hostel whereas Nyokabi and Lukandu (2012) examined the students' satisfaction level found that the students were dissatisfied with the internet services in the hostel. Argueta and Brown (2009) were also of a similar opinion that the international student's housing satisfaction level found that not all the students were satisfied with the fire safety services prevailing in the hostel. Nimako and Bondinuba (2013) evaluated that the students were dissatisfied with the services provided in the hostel including security services. Suki and Chowdhury (2015) confirmed that the students were satisfied with the existing hostel facilities, and they never faced water problems leading them to concentrate peacefully in their studies. Ajayi, Nwous, and Ajani (2015) revealed that the students were dissatisfied with the adequacy of cleanliness and the facilities such as the laundry, bathroom, and toilet facilities due to the distance from the rooms. Bashir, Sarki, and Samidi (2012) considered "student accommodation" as one of the adequate facilities to be provided by the higher educational institutions, and suitable hostel facilities should be provided with security, cooperation, responsible citizenship, intellectual stimulation, inspiration, and mutual composition. Mushtaq and Khan (2012) found that the students' performance improved when the management provided good learning facilities to the students living in the hostel. Ekejiube (2015) found out implications for setting policies and practices regarding the planning and designing of the hostel and the related facilities. Ojo, Chibuzor, Ugochukwu Chukwukelua, and Abidemi (2015) found that there is an association between roommate relationships and students' academic performance. Arshad, Zaidi, and Mahmood (2015) confirmed that there was a relationship between academic performance and self-esteem. Hamed, Miskey, and Sami (2015) found that the effect of the sleeping pattern is an important aspect and has an impact on the academic performance of the students. Lowry, Dean, and Manders (2010) confirmed the same through examining the relationship between gradepoint average and sleep and thus the need for a good sleeping facility. Mehmood and Taswir (2013) examined the effectiveness of using social tools in acquiring knowledge and emphasized that in modern days students are inclined to use social websites a lot.

\section{Research Methodology}

219 respondents were for this study were chosen on a simple random sampling basis among the students both girls and boys from Sohar University who were living in the hostel outside side the campus. The data was collected through a well-defined questionnaire. The collected data was compiled, processed, and analyzed using the Statistical Package for Social Sciences (SPSS). Chi-square tests, ANOVA, and KolmogorovSmirnov tests were used to bring out the findings. 
Findings

Table.1 Demographic details of the respondents

\begin{tabular}{|c|c|c|c|}
\hline Characteristics & & Freq. & $\%$ \\
\hline \multirow{2}{*}{ Gender } & Male & 55 & 25.1 \\
\hline & Female & 164 & 74.9 \\
\hline \multirow{4}{*}{ Age } & $18-19$ years & 107 & 48.9 \\
\hline & $22-24$ years & 94 & 42.9 \\
\hline & $25-27$ years & 13 & 5.9 \\
\hline & $>28$ years & 5 & 2.3 \\
\hline \multirow{2}{*}{ Marital Status } & Single & 194 & 88.6 \\
\hline & Married & 25 & 11.4 \\
\hline \multirow{2}{*}{ Employed } & Working & 20 & 9.1 \\
\hline & Not working & 199 & 90.9 \\
\hline \multirow{7}{*}{ Faculty } & Business & 82 & 24.2 \\
\hline & Engineering & 45 & 27.9 \\
\hline & IT & 82 & 22.8 \\
\hline & Eng.Lang.studies & 9 & 15.5 \\
\hline & GFP & 37 & 6.8 \\
\hline & Law & 4 & 1.8 \\
\hline & Others & 2 & 9 \\
\hline \multirow{5}{*}{ Level } & Level 1 & 55 & 25.1 \\
\hline & Level 2 & 45 & 20.5 \\
\hline & Level 3 & 25 & 11.4 \\
\hline & Level 4 & 55 & 25.1 \\
\hline & GFP & 39 & $\overline{17.8}$ \\
\hline \multirow{2}{*}{$\begin{array}{l}\text { Living in the hostel during last } \\
\text { semester }\end{array}$} & Yes & 170 & 77.6 \\
\hline & No & 49 & 22.4 \\
\hline \multirow{4}{*}{ How long living in the hostel } & $<1$ year & 74 & 33.8 \\
\hline & $1-2$ years & 80 & 36.5 \\
\hline & $3-5$ years & 54 & 24.7 \\
\hline & $>5$ years & 11 & 5.0 \\
\hline \multirow{5}{*}{ GPA during last year } & $1.5-2$ & 33 & 15.1 \\
\hline & $2-2.5$ & 81 & 37.0 \\
\hline & $2.5-3$ & 65 & 29.7 \\
\hline & $3-3.5$ & 32 & 14.6 \\
\hline & $3.5-4$ & 8 & 3.7 \\
\hline \multirow{5}{*}{ GPA during last semester } & $1.5-2$ & 33 & 15.1 \\
\hline & $2-2.5$ & 70 & 32.0 \\
\hline & $2.5-3$ & 76 & 34.7 \\
\hline & $3-3.5$ & 31 & 14.2 \\
\hline & $3.5-4$ & 9 & 4.1 \\
\hline \multirow[b]{2}{*}{ GPA Increased/ decreased } & Yes, increased & 75 & 34.2 \\
\hline & No, decreased & 43 & 19.6 \\
\hline
\end{tabular}




\begin{tabular}{|c|c|c|c|}
\hline & Remains the same & 101 & 46.1 \\
\hline \multirow[t]{4}{*}{ Hours of study per day } & $<1$ hour & 33 & 15.1 \\
\hline & $1-2$ hours & 74 & 33.8 \\
\hline & $2-3$ hours & 53 & 24.2 \\
\hline & $>3$ hours & 59 & 26.2 \\
\hline \multirow{4}{*}{$\begin{array}{l}\text { Hours of study per day in the } \\
\text { hostel }\end{array}$} & $<1$ hour & 50 & 22.8 \\
\hline & $1-2$ hours & 65 & 29.7 \\
\hline & $2-3$ hours & 40 & 18.3 \\
\hline & $>3$ hours & 64 & 29.2 \\
\hline \multirow{4}{*}{$\begin{array}{l}\text { Hours of study outside the } \\
\text { hostel }\end{array}$} & $<1$ hour & 66 & 30.1 \\
\hline & $1-2$ hours & 64 & 29.2 \\
\hline & $2-3$ hours & 44 & 20.1 \\
\hline & $>3$ hours & 45 & 20.5 \\
\hline \multirow[t]{4}{*}{ Hours of study in the library } & $<1$ hour & 102 & 46.6 \\
\hline & $1-2$ hours & 82 & 37.4 \\
\hline & $2-3$ hours & 23 & 10.5 \\
\hline & $>3$ hours & 12 & 5.5 \\
\hline \multirow{2}{*}{$\begin{array}{l}\text { Preferred study place in the } \\
\text { hostel }\end{array}$} & In my room & 169 & 77.2 \\
\hline & In the study room & 50 & 22.8 \\
\hline \multirow{3}{*}{$\begin{array}{l}\text { Preferred study place outside } \\
\text { the hostel }\end{array}$} & In-library & 100 & 45.7 \\
\hline & In classroom & 54 & 24.7 \\
\hline & In the open space & 65 & 29.7 \\
\hline
\end{tabular}

Source: Questionnaire

From the above table no.1, the demographic details of the respondents are observed.

Table.2 Room facilities

\begin{tabular}{|c|c|c|c|c|c|c|c|c|c|}
\hline$\#$ & $\begin{array}{c}\text { Statemen } \\
t\end{array}$ & SD & $\mathbf{D}$ & $\mathbf{N}$ & $\mathbf{A}$ & $\mathbf{S A}$ & $\begin{array}{c}\mathrm{K}-\mathrm{S} \\
\text { value }\end{array}$ & $\begin{array}{c}\text { Chi } \\
\text { Square }\end{array}$ & $\begin{array}{c}\mathbf{p} \\
\text { value }\end{array}$ \\
\hline 1 & Room is peaceful to study & $\begin{array}{c}12 \\
5.5 \%\end{array}$ & $\begin{array}{c}13 \\
5.9 \%\end{array}$ & $\begin{array}{c}56 \\
25.6 \%\end{array}$ & $\begin{array}{c}86 \\
39.3 \%\end{array}$ & $\begin{array}{c}52 \\
23.7 \%\end{array}$ & 7.39 & \multirow{6}{*}{152.521} & \multirow{6}{*}{.000} \\
\hline 2 & Room lighting is good to study & $\begin{array}{c}11 \\
5.0 \%\end{array}$ & $\begin{array}{c}17 \\
7.8 \%\end{array}$ & $\begin{array}{c}44 \\
20.1 \%\end{array}$ & $\begin{array}{c}105 \\
47.9 \%\end{array}$ & $\begin{array}{c}42 \\
19.2 \%\end{array}$ & 7.29 & & \\
\hline 3 & Air condition is comfortable to study & $\begin{array}{c}16 \\
7.3 \%\end{array}$ & $\begin{array}{c}14 \\
6.4 \%\end{array}$ & $\begin{array}{c}44 \\
20.1 \%\end{array}$ & $\begin{array}{c}93 \\
42.5 \%\end{array}$ & $\begin{array}{c}52 \\
23.7 \%\end{array}$ & 7.38 & & \\
\hline 4 & $\begin{array}{l}\text { The location of the room gives a } \\
\text { good environment to study }\end{array}$ & $\begin{array}{c}20 \\
9.1 \%\end{array}$ & $\begin{array}{c}34 \\
15.5 \%\end{array}$ & $\begin{array}{c}60 \\
27.4 \%\end{array}$ & $\begin{array}{c}78 \\
35.6 \%\end{array}$ & $\begin{array}{c}27 \\
12.3 \%\end{array}$ & 5.93 & & \\
\hline 5 & $\begin{array}{l}\text { Refrigerator available in the kitchen } \\
\text { is useful as we need not go out } \\
\text { wasting our time of study }\end{array}$ & $\begin{array}{c}31 \\
14.2 \%\end{array}$ & $\begin{array}{c}30 \\
13.7 \%\end{array}$ & $\begin{array}{c}59 \\
26.9 \%\end{array}$ & $\begin{array}{c}61 \\
27.9 \%\end{array}$ & $\begin{array}{c}38 \\
17.4 \%\end{array}$ & 5.84 & & \\
\hline 6 & $\begin{array}{l}\text { Bathroom is available in my room } \\
\text { which helps me save my time } \\
\text { of study }\end{array}$ & $\begin{array}{c}34 \\
15.5 \%\end{array}$ & $\begin{array}{c}34 \\
15.5 \%\end{array}$ & $\begin{array}{c}36 \\
16.4 \%\end{array}$ & $\begin{array}{c}67 \\
30.6 \%\end{array}$ & $\begin{array}{c}48 \\
21.9 \%\end{array}$ & 6.20 & & \\
\hline
\end{tabular}




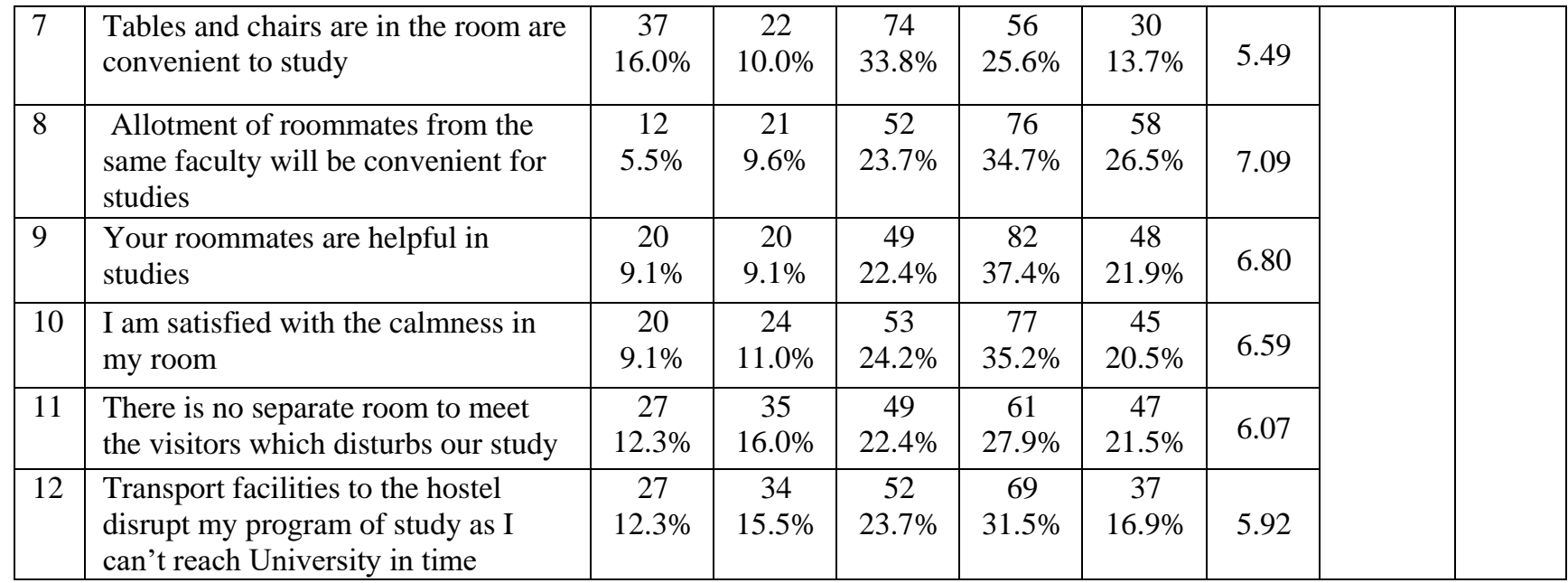

Null hypothesis: There is no relationship between the room facilities and the choices of the respondents.

From the above table No.2, it is observed that p-value is less than 0.05 i.e. the null hypothesis gets rejected. Therefore, comparing the K-S values obtained from the Kolmogorov-Smirnov test, it is observed that 'Room is peaceful to study' ranks first, followed by 'Air-condition is comfortable to study' and 'Room lighting is good to study.'

Table.3 Safety services

\begin{tabular}{|c|c|c|c|c|c|c|c|c|c|}
\hline \# & Statement & SD & D & $\mathbf{N}$ & $\mathbf{A}$ & SA & $\begin{array}{c}\text { K-S } \\
\text { value }\end{array}$ & $\begin{array}{c}\text { Chi- } \\
\text { Square }\end{array}$ & $\begin{array}{c}\text { p- } \\
\text { Value }\end{array}$ \\
\hline 1 & $\begin{array}{l}\text { Hostel is secured with guards, } \\
\text { so there is no fear of studying } \\
\text { anywhere in the hostel }\end{array}$ & $\begin{array}{c}26 \\
11.9 \%\end{array}$ & $\begin{array}{c}14 \\
6.4 \%\end{array}$ & $\begin{array}{c}38 \\
17.4 \%\end{array}$ & $\begin{array}{c}79 \\
36.1 \%\end{array}$ & $\begin{array}{c}62 \\
28.3 \%\end{array}$ & 5.23 & \multirow{9}{*}{165.201} & \multirow{9}{*}{.000} \\
\hline 2 & $\begin{array}{l}\text { The hostel is very much safer } \\
\text { as we can loiter in the corridor } \\
\text { independently }\end{array}$ & $\begin{array}{c}9 \\
4.1 \%\end{array}$ & $\begin{array}{c}10 \\
4.6 \%\end{array}$ & $\begin{array}{c}41 \\
18.7 \%\end{array}$ & $\begin{array}{c}100 \\
45.7 \%\end{array}$ & $\begin{array}{c}59 \\
26.9 \%\end{array}$ & 5.69 & & \\
\hline 3 & $\begin{array}{l}\text { Peaceful sleep without any } \\
\text { fear of safety measure makes } \\
\text { me energetic to concentrate on } \\
\text { my studies }\end{array}$ & $\begin{array}{c}10 \\
4.6 \%\end{array}$ & $\begin{array}{c}14 \\
6.4 \%\end{array}$ & $\begin{array}{c}43 \\
19.6 \%\end{array}$ & $\begin{array}{c}99 \\
45.2 \%\end{array}$ & $\begin{array}{c}53 \\
24.2 \%\end{array}$ & 5.50 & & \\
\hline 4 & $\begin{array}{l}\text { Recreation facilities such as } \\
\text { TV etc. available in the hostel } \\
\text { makes me energetic to } \\
\text { continue my studies }\end{array}$ & $\begin{array}{c}24 \\
11.0 \%\end{array}$ & $\begin{array}{c}23 \\
10.5 \%\end{array}$ & $\begin{array}{c}59 \\
26.9 \%\end{array}$ & $\begin{array}{c}71 \\
32.4 \%\end{array}$ & $\begin{array}{c}42 \\
19.2 \%\end{array}$ & 4.65 & & \\
\hline 5 & $\begin{array}{l}\text { Electricity equipment in the } \\
\text { the hostel is safer and never } \\
\text { disrupted our studies }\end{array}$ & $\begin{array}{c}11 \\
5.0 \%\end{array}$ & $\begin{array}{c}13 \\
5.9 \%\end{array}$ & $\begin{array}{c}57 \\
26.0 \%\end{array}$ & $\begin{array}{c}98 \\
44.7 \%\end{array}$ & $\begin{array}{c}40 \\
18.3 \%\end{array}$ & 5.11 & & \\
\hline 6 & $\begin{array}{l}\text { All kinds of safety equipment } \\
\text { such as smoke, fire blanket } \\
\text { prevail in the hostel never } \\
\text { disrupted our studies }\end{array}$ & $\begin{array}{c}18 \\
8.2 \%\end{array}$ & $\begin{array}{c}19 \\
8.7 \%\end{array}$ & $\begin{array}{c}61 \\
27.9 \%\end{array}$ & $\begin{array}{c}78 \\
35.6 \%\end{array}$ & $\begin{array}{c}43 \\
19.6 \%\end{array}$ & 4.77 & & \\
\hline 7 & $\begin{array}{l}\text { There is no camera available in } \\
\text { the hostel to safeguard our } \\
\text { study materials or belonging } \\
\text { from being stolen }\end{array}$ & $\begin{array}{c}35 \\
16.0 \%\end{array}$ & $\begin{array}{c}36 \\
16.4 \%\end{array}$ & $\begin{array}{c}56 \\
25.6 \%\end{array}$ & $\begin{array}{c}52 \\
23.7 \%\end{array}$ & $\begin{array}{c}40 \\
18.3 \%\end{array}$ & 4.18 & & \\
\hline 8 & $\begin{array}{l}\text { Female securities are not } \\
\text { available in the hostel which is } \\
\text { inconvenient - disturbs our } \\
\text { mood to study }\end{array}$ & $\begin{array}{c}31 \\
14.2 \%\end{array}$ & $\begin{array}{c}39 \\
17.8 \%\end{array}$ & $\begin{array}{c}50 \\
22.8 \%\end{array}$ & $\begin{array}{c}60 \\
27.4 \%\end{array}$ & $\begin{array}{c}39 \\
17.8 \%\end{array}$ & 4.22 & & \\
\hline 9 & $\begin{array}{l}\text { My books and materials are } \\
\text { safe in my room }\end{array}$ & $\begin{array}{c}13 \\
5.9 \%\end{array}$ & $\begin{array}{c}12 \\
5.5 \%\end{array}$ & $\begin{array}{c}44 \\
20.1 \%\end{array}$ & $\begin{array}{c}83 \\
37.9 \%\end{array}$ & $\begin{array}{c}67 \\
30.6 \%\end{array}$ & 5.64 & & \\
\hline
\end{tabular}


Null hypothesis: There is no relationship between the Safety services and the choices of the respondents.

From the above table No.3, it is observed that p-value is less than 0.05 i.e. the null hypothesis gets rejected. Therefore, comparing the K-S values obtained from Kolmogorov-Smirnov test, it is observed from the choice of the respondents that 'The hostel is very much safer as we can loiter in the corridor freely independently' ranks first followed by 'Books and materials are safe in my room,' and 'Peaceful sleep without any fear of safety measure makes me energetic to concentrate on my studies'.

Table.4 Communication services

\begin{tabular}{|c|c|c|c|c|c|c|c|c|c|}
\hline \# & Statement & SD & D & $\mathbf{N}$ & $\mathbf{A}$ & SA & $\begin{array}{c}\mathrm{K}-\mathrm{S} \\
\text { value }\end{array}$ & $\begin{array}{l}\text { Chi- } \\
\text { Square }\end{array}$ & $\begin{array}{c}\text { p- } \\
\text { value }\end{array}$ \\
\hline 1 & $\begin{array}{l}\text { Internet facilities available in the } \\
\text { hostel is not helpful to access study } \\
\text { materials }\end{array}$ & $\begin{array}{c}17 \\
7.8 \%\end{array}$ & $\begin{array}{c}36 \\
16.4 \%\end{array}$ & $\begin{array}{c}54 \\
24.7 \%\end{array}$ & $\begin{array}{c}53 \\
24.2 \%\end{array}$ & $\begin{array}{c}59 \\
26.9 \%\end{array}$ & 5.13 & \multirow{10}{*}{134.288} & \multirow{10}{*}{.000} \\
\hline 2 & $\begin{array}{l}\text { Wi-Fi connection speed is slow to } \\
\text { access study materials }\end{array}$ & $\begin{array}{c}12 \\
5.5 \%\end{array}$ & $\begin{array}{c}18 \\
8.2 \%\end{array}$ & $\begin{array}{c}58 \\
26.5 \%\end{array}$ & $\begin{array}{c}61 \\
27.9 \%\end{array}$ & $\begin{array}{c}70 \\
32.0 \%\end{array}$ & 5.80 & & \\
\hline 3 & $\begin{array}{l}\text { Wi-Fi connection in the hostel is } \\
\text { not helpful to perform my projects }\end{array}$ & $\begin{array}{c}11 \\
5.0 \% \\
\end{array}$ & $\begin{array}{c}29 \\
13.2 \% \\
\end{array}$ & $\begin{array}{c}54 \\
24.7 \%\end{array}$ & $\begin{array}{c}58 \\
26.5 \%\end{array}$ & $\begin{array}{c}67 \\
30.6 \%\end{array}$ & 5.61 & & \\
\hline 4 & $\begin{array}{l}\text { Wi-Fi connection hangs up often } \\
\text { during studies }\end{array}$ & $\begin{array}{l}9 \\
4.1 \%\end{array}$ & $\begin{array}{c}26 \\
11.9 \%\end{array}$ & $\begin{array}{c}51 \\
23.3 \%\end{array}$ & $\begin{array}{c}73 \\
33.3 \%\end{array}$ & $\begin{array}{c}60 \\
27.4 \%\end{array}$ & 5.66 & & \\
\hline 5 & $\begin{array}{l}\text { Wi-Fi not helpful to download any } \\
\text { information in relates to my studies }\end{array}$ & $\begin{array}{c}11 \\
5.0 \%\end{array}$ & $\begin{array}{c}22 \\
10.0 \%\end{array}$ & $\begin{array}{c}49 \\
22.4 \%\end{array}$ & $\begin{array}{c}78 \\
35.6 \%\end{array}$ & $\begin{array}{c}59 \\
26.9 \%\end{array}$ & 5.84 & & \\
\hline 6 & $\begin{array}{l}\text { Social network diverts my } \\
\text { attention from studies }\end{array}$ & $\begin{array}{c}11 \\
5.0 \%\end{array}$ & $\begin{array}{c}29 \\
13.2 \%\end{array}$ & $\begin{array}{c}72 \\
32.9 \%\end{array}$ & $\begin{array}{c}59 \\
26.9 \%\end{array}$ & $\begin{array}{c}48 \\
21.9 \%\end{array}$ & & & \\
\hline 7 & $\begin{array}{l}\text { Need a laptop in the hostel to } \\
\text { access Wi-Fi to download study } \\
\text { materials }\end{array}$ & $\begin{array}{c}11 \\
5.0 \%\end{array}$ & $\begin{array}{c}21 \\
9.6 \%\end{array}$ & $\begin{array}{c}37 \\
16.9 \%\end{array}$ & $\begin{array}{c}80 \\
36.5 \%\end{array}$ & $\begin{array}{c}70 \\
32.0 \%\end{array}$ & 6.14 & & \\
\hline 8 & $\begin{array}{l}\text { In case of disturbance to study, I } \\
\text { am not able to contact the } \\
\text { supervisor }\end{array}$ & $\begin{array}{c}18 \\
8.2 \%\end{array}$ & $\begin{array}{c}19 \\
8.7 \%\end{array}$ & $\begin{array}{c}72 \\
32.9 \%\end{array}$ & $\begin{array}{c}60 \\
27.4 \%\end{array}$ & $\begin{array}{c}50 \\
22.8 \%\end{array}$ & 5.34 & & \\
\hline 9 & $\begin{array}{l}\text { There is no periodic maintenance } \\
\text { of Wi-Fi in the hostel disturbs my } \\
\text { project works }\end{array}$ & $\begin{array}{c}17 \\
7.8 \%\end{array}$ & $\begin{array}{c}36 \\
16.4 \%\end{array}$ & $\begin{array}{c}58 \\
26.5 \%\end{array}$ & $\begin{array}{c}65 \\
29.7 \%\end{array}$ & $\begin{array}{c}43 \\
19.6 \%\end{array}$ & 5.03 & & \\
\hline 10 & $\begin{array}{l}\text { In case of emergency, no telephone } \\
\text { facilities available to contact my } \\
\text { family }\end{array}$ & $\begin{array}{c}23 \\
10.5 \%\end{array}$ & $\begin{array}{c}23 \\
10.5 \%\end{array}$ & $\begin{array}{c}56 \\
25.6 \%\end{array}$ & $\begin{array}{c}72 \\
32.9 \%\end{array}$ & $\begin{array}{c}45 \\
20.5 \%\end{array}$ & 5.26 & & \\
\hline
\end{tabular}

Null hypothesis: There is no relationship between the Communication services and the choices of the respondents.

From the above table No.4, it is observed that p-value is less than 0.05 i.e. the null hypothesis gets rejected. Therefore, comparing the K-S values obtained from Kolmogorov-Smirnov test, it is observed from the choice of the respondents that each 'Need a laptop in the hostel to access Wi-Fi to download study materials' ranks first followed by 'Wi-Fi not helpful to download any information in relates to my studies' and 'Wi-Fi connection speed is slow to access study materials'. 
Table.5 Family support

\begin{tabular}{|c|c|c|c|c|c|c|c|c|c|}
\hline$\#$ & Statement & SD & D & $\mathbf{N}$ & $\mathbf{A}$ & SA & $\begin{array}{c}\text { K-S } \\
\text { value }\end{array}$ & $\begin{array}{c}\text { Chi } \\
\text { Square }\end{array}$ & $\begin{array}{c}\mathbf{P} \\
\text { value }\end{array}$ \\
\hline 1 & $\begin{array}{l}\text { My family prefers me to stay } \\
\text { in the hostel }\end{array}$ & $\begin{array}{c}25 \\
11.4 \%\end{array}$ & $\begin{array}{c}21 \\
9.6 \%\end{array}$ & $\begin{array}{c}48 \\
21.9 \%\end{array}$ & $\begin{array}{c}73 \\
33.3 \%\end{array}$ & $\begin{array}{c}52 \\
23.7 \%\end{array}$ & 5.10 & \multirow{10}{*}{136.155} & \multirow{10}{*}{.000} \\
\hline 2 & $\begin{array}{l}\text { Staying in the hostel, I do not } \\
\text { worry about my family and } \\
\text { concentrate on my studies }\end{array}$ & $\begin{array}{c}23 \\
10.5 \%\end{array}$ & $\begin{array}{c}23 \\
10.5 \%\end{array}$ & $\begin{array}{c}47 \\
21.5 \%\end{array}$ & $\begin{array}{c}70 \\
32.0 \%\end{array}$ & $\begin{array}{c}56 \\
25.6 \%\end{array}$ & 5.13 & & \\
\hline 3 & $\begin{array}{l}\text { Being free-minded and without } \\
\text { family commitment, I } \\
\text { concentrate in my studies }\end{array}$ & $\begin{array}{c}15 \\
6.8 \%\end{array}$ & $\begin{array}{c}25 \\
11.4 \%\end{array}$ & $\begin{array}{c}61 \\
27.9 \%\end{array}$ & $\begin{array}{c}74 \\
33.8 \%\end{array}$ & $\begin{array}{c}44 \\
20.1 \%\end{array}$ & 5.02 & & \\
\hline 4 & $\begin{array}{l}\text { I can perform well in my } \\
\text { studies when I am away from } \\
\text { my family }\end{array}$ & $\begin{array}{c}22 \\
10.0 \%\end{array}$ & $\begin{array}{c}33 \\
15.1 \%\end{array}$ & $\begin{array}{c}62 \\
28.3 \%\end{array}$ & $\begin{array}{c}58 \\
26.5 \%\end{array}$ & $\begin{array}{c}44 \\
20.1 \%\end{array}$ & 4.68 & & \\
\hline 5 & $\begin{array}{l}\text { I do not get family } \\
\text { disturbances through phone } \\
\text { calls, and so I perform well in } \\
\text { studies }\end{array}$ & $\begin{array}{c}17 \\
7.8 \%\end{array}$ & $\begin{array}{c}31 \\
14.2 \%\end{array}$ & $\begin{array}{c}50 \\
22.8 \%\end{array}$ & $\begin{array}{c}72 \\
32.9 \%\end{array}$ & $\begin{array}{c}49 \\
22.4 \%\end{array}$ & 5.09 & & \\
\hline 6 & $\begin{array}{l}\text { Money issues are handled by } \\
\text { my family so that I concentrate } \\
\text { on studies without financial } \\
\text { commitment }\end{array}$ & $\begin{array}{c}7 \\
3.2 \%\end{array}$ & $\begin{array}{c}27 \\
12.3 \%\end{array}$ & $\begin{array}{c}48 \\
21.9 \%\end{array}$ & $\begin{array}{c}69 \\
31.5\end{array}$ & $\begin{array}{c}68 \\
31.1 \%\end{array}$ & 5.73 & & \\
\hline 7 & $\begin{array}{l}\text { My family runs to me if any } \\
\text { problem arises in the hostel } \\
\text { and frees me to concentrate on } \\
\text { my studies }\end{array}$ & $\begin{array}{c}10 \\
4.6 \%\end{array}$ & $\begin{array}{c}12 \\
5.5 \%\end{array}$ & $\begin{array}{c}35 \\
16.0 \%\end{array}$ & $\begin{array}{c}76 \\
34.7 \%\end{array}$ & $\begin{array}{c}89 \\
39.3 \%\end{array}$ & 6.33 & & \\
\hline 8 & $\begin{array}{l}\text { My family comes to my rescue } \\
\text { whenever I find any } \\
\text { transportation problem }\end{array}$ & $\begin{array}{c}9 \\
4.1 \%\end{array}$ & $\begin{array}{c}17 \\
7.8 \%\end{array}$ & $\begin{array}{c}46 \\
21.0\end{array}$ & $\begin{array}{c}61 \\
27.9 \%\end{array}$ & $\begin{array}{c}86 \\
39.3 \%\end{array}$ & 6.08 & & \\
\hline 9 & $\begin{array}{l}\text { Family does shopping for me } \\
\text { to avoid wasting my study time }\end{array}$ & $\begin{array}{c}13 \\
5.9 \%\end{array}$ & $\begin{array}{c}15 \\
6.8 \%\end{array}$ & $\begin{array}{c}49 \\
22.4 \%\end{array}$ & $\begin{array}{c}81 \\
37.0 \%\end{array}$ & $\begin{array}{c}61 \\
27.9 \%\end{array}$ & 5.54 & & \\
\hline 10 & $\begin{array}{l}\text { My parents give moral support } \\
\text { and advice through religious } \\
\text { practices to perform well in the } \\
\text { exams }\end{array}$ & $\begin{array}{c}12 \\
5.5 \%\end{array}$ & $\begin{array}{c}18 \\
8.2 \%\end{array}$ & $\begin{array}{c}30 \\
13.7 \%\end{array}$ & $\begin{array}{c}70 \\
32.0 \%\end{array}$ & $\begin{array}{c}89 \\
40.6 \%\end{array}$ & 6.29 & & \\
\hline
\end{tabular}

Null hypothesis: There is no relationship between the Family support and the choices of the respondents.

From the above table, it is observed that p-value is less than 0.05 i.e. the null hypothesis gets rejected. Therefore, comparing the K-S values obtained from Kolmogorov-Smirnov test, it is observed that 'Family runs to me if any problem arises in the hostel and frees me to concentrate in my studies' ranks first followed by 'My parents give moral support and advice through religious practices to perform well in the exams' and each 'My family comes to my rescue whenever I find any transportation problem'.

Table.6 Study Convenience

\begin{tabular}{|c|c|c|c|c|c|c|c|c|c|}
\hline$\#$ & Statement & SD & $\bar{D}$ & $\mathbf{N}$ & $\overline{\mathbf{A}}$ & $\overline{\text { SA }}$ & $\begin{array}{c}\text { K-S } \\
\text { value }\end{array}$ & $\begin{array}{c}\text { Chi } \\
\text { Square }\end{array}$ & $\begin{array}{c}\mathbf{P} \\
\text { value }\end{array}$ \\
\hline 1 & $\begin{array}{l}\text { Being in the hostel, I can } \\
\text { prepare well for the tests and } \\
\text { examinations }\end{array}$ & $\begin{array}{c}18 \\
8.2 \%\end{array}$ & $\begin{array}{c}12 \\
5.5 \%\end{array}$ & $\begin{array}{c}25 \\
11.4 \%\end{array}$ & $\begin{array}{c}86 \\
39.3 \%\end{array}$ & $\begin{array}{c}78 \\
35.6 \%\end{array}$ & 8.25 & \multirow{3}{*}{116.187} & \multirow{3}{*}{.000} \\
\hline 2 & $\begin{array}{l}\text { I prefer not to sleep the night } \\
\text { before the examination date }\end{array}$ & $\begin{array}{c}46 \\
21.0 \%\end{array}$ & $\begin{array}{c}35 \\
16.0 \%\end{array}$ & $\begin{array}{c}39 \\
17.8 \%\end{array}$ & $\begin{array}{c}65 \\
29.7 \%\end{array}$ & $\begin{array}{c}34 \\
15.5 \%\end{array}$ & 5.39 & & \\
\hline 3 & $\begin{array}{l}\text { Being in the hostel, I could } \\
\text { concentrate well in my studies }\end{array}$ & $\begin{array}{c}11 \\
5.0 \%\end{array}$ & $\begin{array}{c}20 \\
9.1 \%\end{array}$ & $\begin{array}{c}54 \\
24.7 \%\end{array}$ & $\begin{array}{c}81 \\
37.0 \%\end{array}$ & $\begin{array}{c}53 \\
24.2 \%\end{array}$ & 7.16 & & \\
\hline
\end{tabular}




\begin{tabular}{|c|c|c|c|c|c|c|c|c|}
\hline 4 & $\begin{array}{l}\text { I do special prayers for } \\
\text { concentration before starting } \\
\text { to study }\end{array}$ & $\begin{array}{c}13 \\
5.9 \%\end{array}$ & $\begin{array}{c}31 \\
14.2 \%\end{array}$ & $\begin{array}{c}56 \\
25.6 \%\end{array}$ & $\begin{array}{c}64 \\
29.2 \%\end{array}$ & $\begin{array}{c}55 \\
25.1 \%\end{array}$ & 6.75 & \\
\hline 5 & $\begin{array}{l}\text { When I am in the hostel, I can } \\
\text { plan well and manage my time } \\
\text { for studies }\end{array}$ & $\begin{array}{c}8 \\
3.7 \%\end{array}$ & $\begin{array}{c}13 \\
5.9 \%\end{array}$ & $\begin{array}{c}53 \\
24.2 \%\end{array}$ & $\begin{array}{c}93 \\
42.9 \%\end{array}$ & $\begin{array}{c}51 \\
23.3 \%\end{array}$ & 7.43 & \\
\hline 6 & $\begin{array}{l}\text { Being in the hostel, I can } \\
\text { manage to allot time for every } \\
\text { subject }\end{array}$ & $\begin{array}{c}12 \\
5.5 \%\end{array}$ & $\begin{array}{l}15 \\
6.8 \%\end{array}$ & $\begin{array}{c}51 \\
23.3 \%\end{array}$ & $\begin{array}{c}93 \\
42.5 \%\end{array}$ & $\begin{array}{c}48 \\
21.9 \%\end{array}$ & 7.20 & \\
\hline 7 & $\begin{array}{l}\text { I use books for my studies in } \\
\text { the hostel }\end{array}$ & $\begin{array}{c}8 \\
3.7 \%\end{array}$ & $\begin{array}{c}22 \\
10.0 \%\end{array}$ & $\begin{array}{c}43 \\
19.6 \%\end{array}$ & $\begin{array}{c}93 \\
42.5 \%\end{array}$ & $\begin{array}{c}53 \\
24.2 \%\end{array}$ & 7.37 & \\
\hline 8 & $\begin{array}{l}\text { When I live in a hostel, I can } \\
\text { take down notes and attend } \\
\text { classes properly }\end{array}$ & $\begin{array}{c}6 \\
2.7 \%\end{array}$ & $\begin{array}{c}15 \\
6.8 \%\end{array}$ & $\begin{array}{c}48 \\
21.9 \%\end{array}$ & $\begin{array}{c}86 \\
39.3 \%\end{array}$ & $\begin{array}{c}64 \\
29.2 \%\end{array}$ & 7.74 & \\
\hline 9 & $\begin{array}{l}\text { Being in the hostel, I will set } \\
\text { self-determined goals while } \\
\text { studying }\end{array}$ & $\begin{array}{c}7 \\
3.2 \%\end{array}$ & $\begin{array}{c}18 \\
8.2 \%\end{array}$ & $\begin{array}{c}45 \\
20.5 \%\end{array}$ & $\begin{array}{c}88 \\
40.2 \%\end{array}$ & $\begin{array}{c}61 \\
27.9 \%\end{array}$ & 7.54 & \\
\hline 10 & $\begin{array}{l}\text { Being in the hostel I do group } \\
\text { studies well }\end{array}$ & $\begin{array}{c}11 \\
5.0 \%\end{array}$ & $\begin{array}{l}20 \\
9.1 \%\end{array}$ & $\begin{array}{c}60 \\
27.4 \%\end{array}$ & $\begin{array}{c}78 \\
35.6 \%\end{array}$ & $\begin{array}{c}50 \\
22.8 \%\end{array}$ & 6.95 & \\
\hline 11 & $\begin{array}{l}\text { I can study alone and do well } \\
\text { in assessments when I am in } \\
\text { the hostel }\end{array}$ & $\begin{array}{c}13 \\
5.9 \%\end{array}$ & $\begin{array}{c}16 \\
7.3 \%\end{array}$ & $\begin{array}{c}53 \\
24.2 \%\end{array}$ & $\begin{array}{c}75 \\
34.2 \%\end{array}$ & $\begin{array}{c}62 \\
28.3 \%\end{array}$ & 7.30 & \\
\hline 12 & $\begin{array}{l}\text { My memory never gets strong } \\
\text { due to hostel }\end{array}$ & $\begin{array}{c}13 \\
5.9 \%\end{array}$ & $\begin{array}{c}30 \\
16.7 \%\end{array}$ & $\begin{array}{c}77 \\
35.2 \%\end{array}$ & $\begin{array}{c}57 \\
26.0 \%\end{array}$ & $\begin{array}{c}42 \\
19.2 \%\end{array}$ & 6.07 & \\
\hline 13 & $\begin{array}{l}\text { I have good memory power } \\
\text { due to living in the hostel }\end{array}$ & $\begin{array}{c}22 \\
10.0 \%\end{array}$ & $\begin{array}{c}28 \\
12.8 \%\end{array}$ & $\begin{array}{c}68 \\
31.1 \%\end{array}$ & $\begin{array}{c}68 \\
31.1 \%\end{array}$ & $\begin{array}{c}33 \\
15.1 \%\end{array}$ & 5.85 & \\
\hline
\end{tabular}

Null hypothesis: There is no relationship between the Study Convenience and the choices of the respondents.

From the above table, it is observed that p-value is less than 0.05 i.e. the null hypothesis gets rejected. Therefore, comparing the K-S values obtained from Kolmogorov-Smirnov test, it is observed that 'Being in the hostel, I can prepare well for the tests and examinations' ranks first followed by 'When I live in the hostel, I can take down notes and attend classes properly' and 'Being in the hostel, I will set self-determined goals while studying'.

Table 7. (a), (b), (c) and (d) Regression Analysis

\section{Variables Entered /Removed ${ }^{a}$}

\begin{tabular}{|c|l|c|c|}
\hline Model & \multicolumn{1}{|c|}{ Variables Entered } & $\begin{array}{c}\text { Variables } \\
\text { Removed }\end{array}$ & Method \\
\hline $\mathbf{1}$ & $\begin{array}{l}\text { Family Support, Communication Services, Room } \\
\text { Facilities } \\
\text { Safety Services }^{\mathrm{b}}\end{array}$ & $\cdot$ & Enter \\
\hline
\end{tabular}

a Dependent Variable: Study Convenience

${ }^{\mathrm{b}}$ All requested variables entered

\section{Model Summary}

\begin{tabular}{|c|c|c|c|c|}
\hline Model & $\mathrm{R}$ & R Square & $\begin{array}{c}\text { Adjusted R } \\
\text { Square }\end{array}$ & $\begin{array}{c}\text { Std. Error of } \\
\text { the Estimate }\end{array}$ \\
\hline 1 & $.506^{\mathrm{a}}$ & .256 & .242 & 6.021 \\
\hline
\end{tabular}

a Predictors: (Constant) Family Support, Communication Services, Room facilities, Safety Services 
ANOVA $^{\mathrm{a}}$

\begin{tabular}{|l|r|r|r|r|r|}
\hline \multicolumn{1}{|c|}{ Model } & \multicolumn{1}{|c|}{$\begin{array}{l}\text { Sum of } \\
\text { squares }\end{array}$} & \multicolumn{1}{c|}{ df } & \multicolumn{1}{c|}{$\begin{array}{c}\text { Mean } \\
\text { Square }\end{array}$} & F & Sig. \\
\hline Regression & 2663.102 & 4 & 665.775 & 18.366 & $.000^{\mathrm{b}}$ \\
Residual & 7757.675 & 214 & 36.251 & & \\
Total & 10420.776 & 218 & & & \\
& & & & & \\
\hline
\end{tabular}

${ }^{a}$ Dependent Variable: Study Convenience

${ }^{b}$ Predictors: (Constant), Family Support, Communication Services,

Room facilities, Safety Services

\section{Coefficients $^{\text {a }}$}

\begin{tabular}{|c|c|c|c|c|c|}
\hline \multirow[t]{2}{*}{ Model } & \multicolumn{2}{|c|}{$\begin{array}{c}\text { Unstandardized } \\
\text { Coefficients }\end{array}$} & \multirow{2}{*}{$\begin{array}{c}\text { Standardized } \\
\text { Coefficients }\end{array}$} & \multirow[b]{2}{*}{$\mathrm{t}$} & \multirow[b]{2}{*}{ Sig. } \\
\hline & B & $\begin{array}{l}\text { Std. } \\
\text { Error }\end{array}$ & & & \\
\hline (Constant) & 20.368 & 3.444 & & 5.914 & .000 \\
\hline Room facilities & .081 & .068 & .083 & 1.180 & .239 \\
\hline Safety services & .059 & .096 & .045 & .620 & .536 \\
\hline Communication Services & .155 & .070 & .136 & 2.202 & .029 \\
\hline Family Support & .438 & .076 & .389 & 5.746 & .000 \\
\hline
\end{tabular}

${ }^{\text {a }}$ Dependent Variable: Study convenience

From the above table No.7 (a), (b), (c), and (d), it can be seen that p-value for the variables Room Facilities and Safety Services are greater than .05. So eliminating these variables, we redo the regression analysis again, and we obtain the following:

Table 8. (a), (b), (c), (d) and (e) Regression Analysis - Revised

\section{Variables Entered /Removed}

\begin{tabular}{|c|l|c|c|}
\hline Model & \multicolumn{1}{|c|}{ Variables Entered } & $\begin{array}{c}\text { Variables } \\
\text { Removed }\end{array}$ & method \\
\hline $\mathbf{1}$ & $\begin{array}{l}\text { Family support } \\
\text { communication services }\end{array}$ & $\cdot$ & Enter \\
\hline
\end{tabular}

${ }^{\text {a }}$ Dependent Variable: Study convenience

${ }^{\mathrm{b}}$ All requested variables entered

\section{Model Summary}

\begin{tabular}{|c|c|c|c|c|}
\hline Model & $\mathrm{R}$ & R Square & $\begin{array}{c}\text { Adjusted R } \\
\text { Square }\end{array}$ & $\begin{array}{c}\text { Std. Error of } \\
\text { the Estimate }\end{array}$ \\
\hline 1 & $.495^{\mathrm{a}}$ & .245 & .238 & 6.033 \\
\hline
\end{tabular}

${ }^{\text {a }}$ Predictors: (constant), Family support, communication services 
ANOVA ${ }^{a}$

\begin{tabular}{|l|c|c|c|c|c|}
\hline \multicolumn{1}{|c|}{ Model } & $\begin{array}{c}\text { Sum of } \\
\text { squares }\end{array}$ & df & Mean Square & F & Sig. \\
\hline Regression & 2557.837 & 2 & 1278.918 & 35.133 & $.000^{\mathrm{b}}$ \\
Residual & 7862.940 & 216 & 36.402 & & \\
Total & 10420.776 & 218 & & & \\
\hline
\end{tabular}

${ }^{\text {a }}$ Dependent Variable: Study convenience

${ }^{\mathrm{b}}$ Predictors: (constant), Family support, communication services

Coefficients a

\begin{tabular}{|c|c|c|c|c|c|}
\hline \multirow[t]{2}{*}{ Model } & \multicolumn{2}{|c|}{$\begin{array}{l}\text { Unstandardized } \\
\text { Coefficients }\end{array}$} & \multirow{2}{*}{$\begin{array}{c}\text { Standardized } \\
\text { Coefficients } \\
\text { Beta }\end{array}$} & \multirow[b]{2}{*}{$\mathrm{t}$} & \multirow[b]{2}{*}{ Sig. } \\
\hline & B & Std.Error & & & \\
\hline (constant) & 23.014 & 3.080 & & 7.473 & .000 \\
\hline $\begin{array}{l}\text { Communication } \\
\text { services }\end{array}$ & .165 & .069 & .145 & 2.373 & .019 \\
\hline Family support & .493 & .069 & .438 & 7.164 & .000 \\
\hline
\end{tabular}

${ }^{a}$ Dependent Variable: Study convenience

The ANOVA table shows that the p-value $<.05$.

Therefore, we obtain the regression equation as follows:

$\mathrm{S} \mathrm{C}=23.014+.165 \mathrm{CS}+.493 \mathrm{FS}$

Where S C is Study Convenience, CS is Communication services, and FS is Family Support.

There is a direct association between Communication Services, Family Support, and Study Convenience. i.e. there is an impact of Communication Services and Family support on Study Convenience.

\section{Results and Discussions}

Most of the respondents are living in the hostel for more than one year (66.2\%). $81.3 \%$ of the respondents reported that their GPA during last semester was more than 2 and this year $84.9 \%$ of respondents reported to be of GPA more than 2. 34.2\% of the respondents reported that their GPA has increased after their hostel stay. It is observed that the study during the stay in the hostel is high compared to their study outside the hostel or at home.

Most of the respondents $(77.2 \%)$ are so comfortable and peaceful to study in their hostel room. The majority of the students also reported that the room lighting is good to study, Air conditioning is comfortable and the room is located in a good environment to study. It was also reported that the furniture provided was also good. Further, they also reported that the bathroom, kitchen facilities comforts their study from wasting their time going outside. But the majority complained that there is no separate room to meet their visitors, disturbs their study. Most of the respondents (48.4\%) reported that the transport facilities to the hostel disrupt their program of study as there is a delay in reaching the University on time.

From the security point of view, most of the respondents $(64.4 \%)$ reported that the hostel is secured with guards and so there is no fear of studying anywhere in the hostel, and thus they have reported that they can loiter in the corridor independently. Most of the respondents (69.7\%) reported that peaceful sleep in the hostel room (without any fear) makes them fresh and energetic to concentrate on their studies, and they have also reported that the relaxation facilities like TV, etc. provided in the common room of the hostel makes them relaxed and energetic to continue their studies. Further, they also reported that the Electricity equipment in the hostel are safer and never disrupted their studies. In general, it was reported that all kinds of safety equipment such as smoke, fire blanket prevail in the hostel never disrupted their studies rather made them free-minded. But most of the respondents $(43.9 \%)$ have reported that there is no camera available in the hostel to safeguard their study materials or belongings being stolen, and it was a major concern that the female securities are not available in the hostel, which they felt as inconvenient - disturbs their mood to study. However, most of the respondents $(58.0 \%)$ reported that their books and materials are safe in their hostel rooms. 
Most of the students (49.1\%) believe that the internet facilities available in the hostel is not helpful to access the study materials and the Wi-Fi connection speed was slow especially the Wi-Fi connection in the hostel was not helpful to perform their project works, and there is no proper periodic maintenance of it. They also reported that the Wi-Fi connection hangs up quite often during their studies and it is also reported that Wi-Fi was not helpful to download any information in relates to studies. Most of the respondents (48.8\%) believe that Social network affects their academic performance by diverting their attention from studies. Most of the respondents (68.5\% agree) demanded a laptop in the hostel to access Wi-Fi to download study materials. Most of the respondents (53.4\%) reported that in the case of emergency, there are no telephone facilities available to contact their families which disturb them, and further it was also reported that in the case of disturbance to study they were not able to contact the hostel supervisors as well.

Most of the respondents $(57.0 \%)$ believe that their family prefers their wards to stay in the hostel and have opined that they do not worry/think about their family and fully concentrate on studies. Most of the respondents $(53.9 \%)$ opined that free-minded, without family commitment makes them perform in their studies well. Most of the respondents $(46.6 \%)$ confirmed that they were able to perform well in studies when they were away from their families and no phone calls from their families. It was also reported that the money related issues are handled by their families so they could concentrate well without financial commitments. Most of the respondents reported that their family comes to their rescue in case any problem arises in a hostel or any transportation issues. It was also reported that their family does shopping for them to avoid wasting study time and the parents reported to give moral support and advice through religious practices to perform well in exams.

Most of the respondents (74.9\%) reported that they prepare well for their tests and examinations and $45.2 \%$ of the respondents preferred not to sleep the night before the examination date. $44.3 \%$ reported that they do special prayers for concentration before starting their study. Most of the students reported that they could plan well and manage their time and allot time for every subject. $46.2 \%$ of the respondents believed that they had good memory power due to living in the hostel. $66.7 \%$ agree reported that they use books for their studies and $68.5 \%$ reported that they are taking down notes and attended the classes properly. Most of the students $(58.4 \%)$ stated that being in the hostel, they can do group studies well whereas $62.5 \%$ reported that they could study alone and do well in their assessments.

Amongst the room facility factors, 'Room is peaceful to study' ranked first, followed by 'Air-condition is comfortable to study' and each 'Room lighting is good to study.'

Amongst the safety services factors 'The hostel is very much safer as we can loiter in the corridor freely independently' ranked first, followed by 'Books and materials are safe in my room, ' and 'Peaceful sleep without any fear of safety measure makes me energetic to concentrate on my studies.'

Amongst the communication services factors, 'Need a laptop in the hostel to access Wi-Fi to download study materials' ranked first, followed by 'Wi-Fi not helpful to download any information in relates to my studies' and 'Wi-Fi connection speed is slow to access study materials.'

Amongst the family support factors, 'Family runs to me if any problem arises in the hostel and frees me to concentrate in my studies' ranked first followed by 'My parents give moral support and advice through religious practices to perform well in the exams' and each 'My family comes to my rescue whenever I find any transportation problem'.

Amongst the study convenience factors, 'Being in the hostel, I can prepare well for the tests and examinations' ranked first followed by 'When I live in the hostel, I can take down notes and attend classes properly' and 'Being in the hostel, I will set self-determined goals while studying'.

\section{Conclusion}

The study shows that the students are satisfied with the room facilities provided in the hostel and also satisfied with the safety services provided in the hostel. But they are not satisfied with the communication services prevailing in the form of Internet services (Wi-Fi) and telephone facilities. It is learned that the internet services are not up to the mark and the connection hangs up most of the time and the speed of the internet connection is also very slow.

The study also throws light on the fact that there exists a linear relationship between the Communication Services, Family Support, and Study Convenience of the students living in a hostel. In other words, the study emphasizes that there is an impact of Communication Services and Family Support on Study Convenience for the students living in a hostel. The same is explained through a model named Communication-Family Support-Convenience (CFSC) model shown below: 


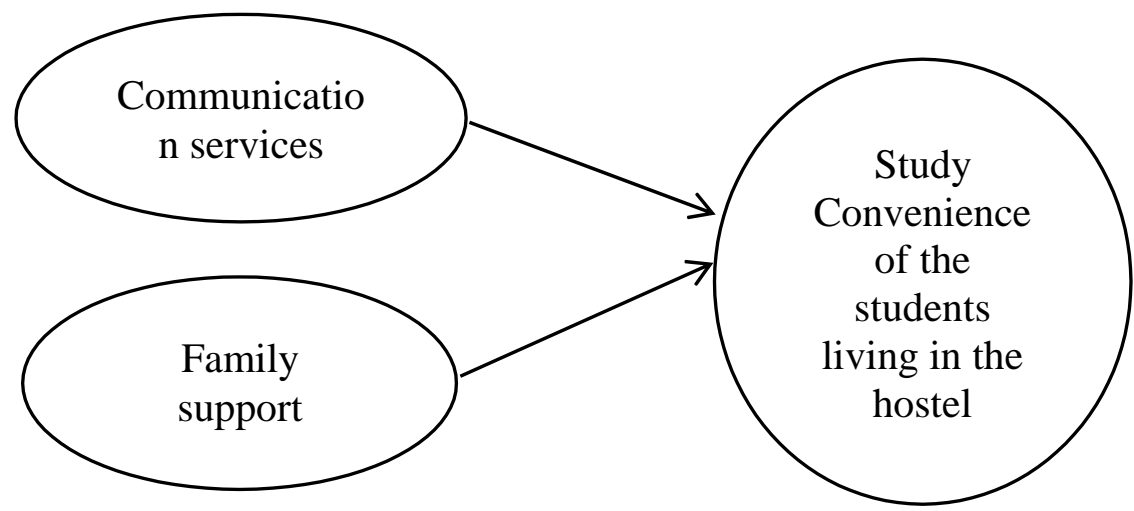

Fig.1 Communication-Family Support-Convenience (CFSC) Model

Though the factors taken into consideration during our study were Room Facility services, Communication Services, Family Support, and Study Convenience, they impact on Study convenience for the students living in the hostel were only due to Communication services and Family Support.

\section{Recommendation}

Thus, the hostel authorities and administration should pay more attention to provide good communication facilities in the hostel, in particular, speedy internet connection could be made available through $\mathrm{Wi}-\mathrm{Fi}$ to convene the students. 


\section{References}

Ajayi, M., Nwosu, A., and Ajani, Y. (2015), "Students Satisfaction with hostel facilities in the Federal University of Technology," European Scientific Journal, Vol.11, issue. 34, pp. 402-415.

Araujo, P. and Murray, J. (2010), "Estimating the Effects of Dormitory Living on Student performance," Center for Applied Economics and Policy Research, Vol. 15, Issue 2, pp.1-15.

Argueta, J. and Brown, N. (2008), "An assessment of fire safety in Australia s international student housing," The Institute of International Education, Vol.13, Issue 33, pp.55-133.

Arshad, M., Zaidi, M. and Mahmood, K. (2015), "Self Esteem \& Academic performance among University students," Journal of Education and practica, Vol.6, issue.1, pp. 156-162.

Bashir, S., Sarki, I.H. and Samidi, J. (2012), "Students' perception of the service quality of Malaysian university hostel accommodation," International Journal of Business and Social Science, Vol.3, issue. 15, pp. 213-222.

Ekejiube, P. (2015), "The running of hostels as auxiliary services in University of Benin: Implications for academic studies," African Educational Research Journal, Vol.3, issue1, pp 51-54.

Hamed, H., Miskey, A., and Sami, R. (2015), "The effect of sleeping pattern on the Academic performance of undergraduate medical students at Ajman University of Science and Technology," IOSR Journal of Pharmacy, Vol.5, Issue 6, pp.22-50.

Iftikar, A. and Ajmal, A. (2015), "Qualitative Study Investigating the Impact of Hostel life," International Journal of Emergency Mental Health and Human Resilience, Vol. 17, Issue 2, pp.511-515.

Kamaruddin, R., Zainal, N. and Aminuddin, Z. (2009), "The Quality of Learning Environment and academic performance from students perception," International Journal of business and management, Vol.4, issue. 4, pp. 171-175.

Khozaei, F., Ayub, N., Hassan, A. and Khozaei, A. (2010), “The factors predicting students' satisfaction with University hostels," Asian Culture and History, Vol. 2, Issue 2, pp. 148-158.

Khurshid, F., Tanveer, A. and Qasmi, F. (2012), "Relationship between Study Habits and Academic Achievement among Hostel living and Day Scholars university students," British Journal of Humanities and social sciences, Vol. 3, Issue 2, pp. 34-42.

Lowry, M., Dean, K. and Manders, K. (2010), “The Link Between Sleep Quantity and Academic Performance for the College Student,” The University Minnesota Undergraduate Journal of Psychology, Vol.3, issue15, pp.16-19.

Mahama, F., Boahen, P.A., Saviour, A. and Tumaku, J. (2010), "Modeling satisfaction factors that predict students' choice of private hostels in a Ghanaian polytechnic," British Journal of Mathematics and Computer Science, Vol.19, issue. 3, pp.453-564.

Mansoor, U. and Ali, M. (2015), "Impact hostel Students satisfaction on their Academic performance in Srilankan Universities," International Symposium, Vol. 9, issue.3, pp. 62-70.

Mehmood, S. and Taswir, T. (2013), "The Effect of Social Networking Site on the Academic performance of students in the College of Applied Sciences," International Journal of Arts and Commerce, Vol. 2, issue1, pp111-125.

Mushtaq, I. and Khan, S. (2012), "Factors Affecting Students' Academic Performance," Global Journal of Management and Business Research, Vol. 12, Issue 9, pp1-7.

Ning, Y. and Chen, J. (2016), "Improving residential satisfaction of university dormitories through postoccupancy evaluation in China: a socio-technical system approach," Sustainability, Vol.8, issue.1050, pp. 117.

Nimako, S. and Bondinuba, F. (2013), "An Empirical Evaluation of Student Accommodation Quality in Higher," European Journal of Business and Social Sciences, Vol. 1, issue.12, pp. 164-1770.

Nyokabi, K. and Lukandu, A. (2012), "Evaluation of the relationship between internet accessibility and students," International Research Journals, Vol.3, issue. 2, pp. 137-144.

Ojo, D., Chibuzor, M., Ugochukwu, O., Chukwukelua, O. and Abidemi, O. (2015), "Effect of college roommate relationships on student development at a private University," Mediterranean Journal of Social Sciences, Vol. 6, issue. 6, pp. 1-11.

Owolabi, B.O. (2015), "The Effects of students' Housing on Academic Performance at the University of Ibadan in Nigerian," International Journal of Scientific \& Engineering Research, Vol. 6, issue 3, pp. 11181132.

Owusu-Acheaw, O. and Larson, A.G. (2015), "Use of social media and its impact on academic performance of Tertiary institution students: A student of Students Koforidua Polytechnic, Ghana," Journal of Education and Practice, Vol. 6, issue. 6, pp. 94-101.

Owusu, M.A. (2014), "Reading habits Among Students and its Effect on Academic performance: A study of students of Koforidua Polytechnic,” Library Philosophy and Practice, Vol.6, issue. 5, pp. 1-22. 
Shakeel, A., Shakeel, S., and Fatima (2015), "Life Satisfaction and Quality of Life among Hostelized and Day Scholar Female Students,” European Journal of Business and Social Sciences, Vol.4, issue. 08, pp. 119127.

Suki, N., Chowdhury, I. (2015), "Students Attitude and Satisfaction living in Sustainable on-Campus Hostels," Malaysian Journal of Business and Economics, Vol. 2, issue. 1, pp. 35-47.

Veena, G. (2015), "Perception and usage of Wi-Fi infrastructure among Students Community," International Journal of Academic Library and Information Science, Academic Research Journals, Vol.3, issue; 11, pp. 336-341, available at http://www.academicresearchjournals.org/IGALIS/Index.htm

Copyright: @ 2020 by the authors. Licensee Global Scientific Publications, Oman.

This work is licensed under a Creative Commons Attribution-ShareAlike 4.0 International License.

This is an open-access journal and the articles published in this journal are distributed under the terms of CC-BY-SA. 